\title{
Design of Water Supply Pipe Networks in NIT Srinagar using EPANET Software
}

\author{
Sandeep Thakur ${ }^{[1]}$, Abhinav Verma ${ }^{[1]}$, VK Angral ${ }^{[1]}$, Dr. M.A. Ahanger ${ }^{[2]}$, \\ ${ }^{[1]}$ B. tech Scholar, Civil Engineering, National Institute of Technology, Srinagar, J\&K, India \\ ${ }^{[2]}$ Professor, Civil Engineering, National Institute of Technology, Srinagar, J\&K, India
}

\begin{abstract}
Water is one of the most vital natural resources for all life on Earth. The availability and quality of water always have played an important part in determining not only where people can live, but also their quality of life. Even though there always has been plenty of fresh water on Earth, water has not always been available when and where it is needed, nor is it always of suitable quality for all uses. Water must be considered as a finite resource that has limits and boundaries to its availability and suitability for use. Water is used for various purposes like commercial ( hotels, restaurants, office buildings, other commercial facilities), domestic (drinking, food preparation, bathing, washing clothes and dishes, flushing toilets, and watering lawns and gardens), industrial( cleaning, transportation, dilution, and cooling in manufacturing facilities), irrigation (freeze protection, chemical application, crop cooling, harvesting, and for the leaching of salt from crop zone). For all this purposes we need to supply water which is done by water distribution system.

In this study pipe network system has been designed for NIT SRINAGAR using EPANET software for 30 years population projection (design period). The design consists of hydraulic design. Population of the campus (including residential and non-residential) with a margin for increase in population has been considered while determining the water demand.
\end{abstract}

\section{INTRODUCTION: -}

Water distribution systems connect consumers to sources of water, using hydraulic components, such as pipes, valves, and reservoirs. The engineer faced with the design of such a system, or of additions to an existing system, has to select the sizes of its components. Also, he has to consider the way in which the operational components, pumps and valves, will be used to supply the required demands with adequate pressures. The network has to perform adequately under varying demand loads, and in the design process, one considers several significant loads: maximum hourly, average daily, low-demand periods during which reservoirs are to be filled, etc. Operational decisions for these loads are essentially part of the design process, since one cannot separate the so-called design decisions, the sizing of components, from the operational decisions; they are two inseparable parts of one problem. This paper presents a method for optimizing the design of a water distribution system: sizing its components and setting the operational decisions for pumps and valves under a number of loading conditions, those which are considered 'typical' or ' critical. ' EPANET can be used in the planning of pipe network systems to meet forecasted demands of the next 20 years or 30 years. For example, the program can be used to develop long term capital-improvement plans for the existing pipe network system. These plans can include staging, sizing, and locating future pipe network and water chlorination facilities. The software can also be used in the development of a main rehabilitation plan or a system-improvement plan. And, a network analysis can provide suggestions and recommendations to prepare for the occurrence of any unusual events.

\section{EXISTING WORK: -}

Pipe network analysis of water distribution systems has evolved from a time-consuming process done infrequently to a quick and easy process done regularly on systems of all sizes. Pipe network analysis initially started early in 1940. Years later, two network analysis programs were introduced by Shamir and Howard (1968) and Epp and Fowler (1970). Both programs used the Newton-Raphson method to linearize the nonlinear mass and energy equations. The major differences between these two programs are:

1. The Shamir-Howard program is based on node-oriented equations, while the

Epp-Fowler program is based on loop-oriented equations.

2. The Shamir-Howard program solves for pressure, demand, and the parameters of pipes and nodes, while the Epp-Fowler program solves only for pressures and flow rates.

\section{Estimation of Water Demand}

\section{IMPLEMENTATION: -}

Assuming consumption of water as per specified by IS Code 1172:1993 as follow:

1. $135 \mathrm{lpcd}$ for hostel

2. 50 lpcd for floating population

3. $150 \mathrm{lpcd}$ for residential buildings

4. $50 \mathrm{lpcd}$ for offices

5. $450 \mathrm{lpcd}$ for medical unit

6. 350 lpcd for medical quater

7. 2 as peak factor 


\begin{tabular}{|c|c|c|c|c|c|c|}
\hline BRANCH & $\begin{array}{l}\text { BTech } \\
\text { Student }\end{array}$ & B & $\begin{array}{l}\text { MTECH } \\
\text { STUDENT } \\
\text { S }\end{array}$ & $\begin{array}{l}\text { MTECH } \\
\text { STUDENTS }\end{array}$ & $\begin{array}{l}\text { Demand } \\
\text { (lpd) } \\
\text { @ 50lpcd }\end{array}$ & $\begin{array}{l}\text { Demand } \\
\text { (lps) }\end{array}$ \\
\hline & $\begin{array}{l}\text { Present } \\
\text { (2014) }\end{array}$ & $\begin{array}{l}\text { Future } \\
\text { estimated }\end{array}$ & $\begin{array}{l}\text { Present } \\
\text { (2014) }\end{array}$ & Future (2014) & & \\
\hline \multirow{4}{*}{$\begin{array}{l}\text { CIVIL } \\
\text { ENGINEERING }\end{array}$} & \multirow[t]{4}{*}{123} & \multirow[t]{4}{*}{185} & $\begin{array}{ll}\text { GE } & 12 \\
\end{array}$ & 27 & \multirow[t]{4}{*}{970} & \multirow{4}{*}{$\begin{array}{l}97000 \mathrm{lpd} \\
=1.123 \mathrm{lps}\end{array}$} \\
\hline & & & $\begin{array}{ll}\text { SE } & 20 \\
\end{array}$ & 35 & & \\
\hline & & & TE 13 & 28 & & \\
\hline & & & WRE 10 & 25 & & \\
\hline $\begin{array}{l}\text { ELECTRICAL } \\
\text { ENGINEERING }\end{array}$ & 78 & 117 & EPE 21 & 36 & 540 & $\begin{array}{l}54000 \mathrm{lpd} \\
=0.625 \mathrm{lps}\end{array}$ \\
\hline $\begin{array}{l}\text { MECHANICAL } \\
\text { ENGINEERING }\end{array}$ & 77 & 116 & $\begin{array}{l}\text { MSD } 20 \\
\text { ITMM } 21\end{array}$ & $\begin{array}{l}35 \\
36 \\
\end{array}$ & 606 & $\begin{array}{l}60600 \mathrm{lpd} \\
=0.701 \mathrm{lps}\end{array}$ \\
\hline $\begin{array}{l}\text { ELECTRONICS } \\
\text { AND } \\
\text { COMMUNICATION }\end{array}$ & 77 & 116 & $\begin{array}{ll}\text { ME } & 11 \\
\text { C\&IT } & 20\end{array}$ & $\begin{array}{l}26 \\
35\end{array}$ & 586 & $\begin{array}{l}58600 \mathrm{lpd} \\
=0.678 \mathrm{lps}\end{array}$ \\
\hline $\begin{array}{l}\text { INFORMATION } \\
\text { TECHNOLOGY }\end{array}$ & 62 & 92 & NA & 20 & 408 & $\begin{array}{l}40800 \mathrm{lpd} \\
=0.472 \mathrm{lps}\end{array}$ \\
\hline $\begin{array}{l}\text { CHEMICAL } \\
\text { ENGINEERING }\end{array}$ & 78 & 117 & CHE 15 & 30 & 528 & $\begin{array}{l}52800 \mathrm{lpd} \\
=0.61 \mathrm{lps}\end{array}$ \\
\hline COMPUTER SCIENCE & 62 & $\checkmark$ & NA & 20 & 408 & $\begin{array}{l}40800 \mathrm{lpd} \\
=0.472 \mathrm{lps}\end{array}$ \\
\hline $\begin{array}{l}\text { METALLURGY } \\
\text { ENGINEERING }\end{array}$ & 78 & 117 & NA & 20 & 508 & $\begin{array}{l}50800 \mathrm{lpd} \\
=0.587 \mathrm{lps}\end{array}$ \\
\hline
\end{tabular}

NA stands for not available *lpd stands for liters per day *lps stands for liters per second *Seat matrix is taken from CCMT and counselling websites.

For hostels:

As hostels are nearly full up to their capacity so future increment is given to them and assume 135 lpcd as water demand with peak factor of 2 as recommended by code IS: 1172:1993.

Table 2: Water demand for Hostels

\begin{tabular}{|c|c|c|c|c|}
\hline $\begin{array}{l}\text { Name of } \\
\text { Hostel }\end{array}$ & No of rooms & Capacity & $\begin{array}{l}\text { Water } \\
\text { Demand } \\
\text { @135lpcd @ } 2 \\
\text { peak factor }\end{array}$ & $\begin{array}{l}\text { Residual Head Required } \\
\text { (in } \mathrm{m} \text { ) }\end{array}$ \\
\hline Jhelum & 300 in 6 blocks & $\begin{array}{l}1 \text { person per room } \\
=300\end{array}$ & $\begin{array}{l}81000 \mathrm{lpd} \\
=0.94 \mathrm{lps}\end{array}$ & 17 \\
\hline Chenab & 99 & $\begin{array}{l}4 \text { persons per room } \\
=396\end{array}$ & $\begin{array}{l}106920 \mathrm{lpd} \\
=1.24 \mathrm{lps}\end{array}$ & 17 \\
\hline Indus & 123 & $\begin{array}{l}4 \text { persons per room } \\
\quad=492\end{array}$ & $\begin{array}{l}132840 \mathrm{lpd} \\
=1.54 \mathrm{lps}\end{array}$ & 17 \\
\hline Tawi & 48 & 4 persons per room $=192$ & $\begin{array}{l}51840 \mathrm{lpd}= \\
0.6 \mathrm{lps}\end{array}$ & 7 \\
\hline Dal & 48 sets & $\begin{array}{l}5 \text { persons per set } \\
\quad=240\end{array}$ & $\begin{array}{l}64800 \mathrm{lpd} \\
=0.75 \mathrm{lps}\end{array}$ & 17 \\
\hline S-Hostel & 72 sets & $\begin{array}{l}5 \text { persons per set } \\
\quad=360\end{array}$ & $\begin{array}{l}97200 \mathrm{lpd} \\
=1.125 \mathrm{lps}\end{array}$ & 17 \\
\hline $\begin{array}{l}\text { M TECH } \\
\text { Hostel }\end{array}$ & $\begin{array}{l}\text { Block 1 } \\
26 \\
\end{array}$ & $\begin{array}{l}3 \text { persons per room } \\
=78\end{array}$ & $\begin{array}{l}21060 \mathrm{lpd} \\
=0.244 \mathrm{lps}\end{array}$ & 12 \\
\hline & $\begin{array}{l}\text { Block } 2 \\
4\end{array}$ & $\begin{array}{l}4 \text { persons per room } \\
=16\end{array}$ & $\begin{array}{l}4320 \mathrm{lpd} \\
=0.05 \mathrm{lps}\end{array}$ & 7 \\
\hline Mega Hostel & \multicolumn{2}{|c|}{$\begin{array}{l}5 \text { storey building with capacity of } \\
1000 \text { persons }\end{array}$} & $\begin{array}{l}270000 \mathrm{lpd} \\
=3.13 \mathrm{lps}\end{array}$ & 27 \\
\hline Girls Hostel & 12 sets & 180 & $\begin{array}{l}48600 \mathrm{lpd}= \\
0.562 \mathrm{lps}\end{array}$ & 17 \\
\hline
\end{tabular}

Total water demand for Hostels $=878580 \mathrm{lpd}=10.169 \mathrm{lps}$

Also, there are two blocks for Pre-fabricated hostel which is built near jhelum hostel. Assuming 135 lpcd with capacity of 400 persons, then water demand will be equal to 1.25 lps. 
Table 3: Water Demand for new blocks

\begin{tabular}{|c|c|c|c|}
\hline BLOCK NO & Specifications of blocks & $\begin{array}{l}\text { Water demand } \\
(50 \text { lpcd for Floating } \\
\text { population and } 150 \text { lpcd for } \\
\text { residential with peak factor } 2)\end{array}$ & $\begin{array}{l}\text { HEAD } \\
\text { REQUIRED }\end{array}$ \\
\hline 1 & $\begin{array}{l}\text { 6nos. 100 Capacity Drawing } \\
\text { Hall With A2 Size Drawing } \\
\text { Board } \\
25 \text { Nos. } 100 \text { Capacity Lecture } \\
\text { Theatre With Stepped Seats } \\
20 \text { Nos. } 40 \text { Capacity } \\
\text { Classrooms On Level Floor, } \\
\text { Computer Centre = } 3 \text { floor } \\
\text { Electrical Engineering (Ext.)..... } \\
1 \text { Floor } \\
\text { Academic } \\
\text { Section/Examination Halls --- } \\
\text {---- } 1 \text { Floor } \\
\text { Deans Offices/ Cafeteria ----- } \\
1 \text { Floor }\end{array}$ & $\begin{array}{l}\text { Floor } 1=0.69 \mathrm{lps} \\
\text { Floor } 2=2.89 \mathrm{lps} \text { Floor } 3=0.93 \\
\text { lps Floor } 4=0.24 \text { lps Floor } 5= \\
0.02 \mathrm{lps} \text { Floor } 6=0.02 \mathrm{lps} \\
\text { Total }=4.79 \mathrm{lps}\end{array}$ & $32 \mathrm{~m}$ \\
\hline 2 & $\begin{array}{l}\text { IT ----- 1 Floor } \\
\text { Computer Science And } \\
\text { Engineering ------ } 1 \text { Floor } \\
\text { Physics } \\
\text { Chemistry ----- } 1 \text { Floor } \\
\text { Maths ---- } 1 / 2 \text { Floor } \\
\text { Humanities ----- } 1 / 2 \text { Floor } \\
\text { Electronics Comm.Enggdept., } \\
\text { CRF Centre ----- } 1 \text { Floor }\end{array}$ & $\begin{array}{l}\text { Floor } 1=0.17 \mathrm{lps} \\
\text { Floor } 2=0.175 \mathrm{lps} \\
\text { Floor } 3=0.21 \mathrm{lps} \\
\text { Floor } 4=0.21 \mathrm{lps} \\
\text { Floor } 5=0.21 \mathrm{lps} \\
\text { Floor } 6=0.24 \mathrm{lps} \\
\text { Total }=1.215 \mathrm{lps}\end{array}$ & $32 \mathrm{~m}$ \\
\hline 3 & $\begin{array}{l}\text { Workshops ------ 1 Floor } \\
\text { Mechanical Engineering } \\
\text { 2floor } \\
\text { Chemical Engineering } \\
\text { Metallurgical Engineering } 1 \text { Floor } \\
\text { Civil Lab /WRMC } \\
\text { Floor }\end{array}$ & $\begin{array}{l}\text { Floor } 1=0.20 \mathrm{lps} \text { Floor } 2-3= \\
0.25 \\
\text { lps } \\
\text { Floor } 4=0.21 \mathrm{lps} \\
\text { Floor } 5=0.21 \mathrm{lps} \\
\text { Floor } 6=0.09 \mathrm{lps} \\
\text { Total }=0.97 \mathrm{lps}\end{array}$ & $32 \mathrm{~m}$ \\
\hline 4 & $\begin{array}{l}\text { Hospital } \\
2 \text { Nos. Banks } \\
\text { ATMs } \\
\text { Telephone Exchange } \\
\text { Shopping Mall } \\
\text { P\&D Stores } \\
\text { Service Centre }\end{array}$ & $\begin{array}{l}\text { Floor } 1=0.07 \mathrm{lps} \\
\text { Floor } 2=0.12 \mathrm{lps} \\
\text { Floor } 3=0.12 \mathrm{lps} \\
\text { Floor } 4=0.12 \mathrm{lps} \\
\text { Floor } 5=0.12 \mathrm{lps} \\
\text { Floor } 6=0.06 \mathrm{lps} \\
\text { Total }=0.61 \mathrm{lps}\end{array}$ & $32 \mathrm{~m}$ \\
\hline 5 & $\begin{array}{l}\text { Girls Hostel With Single } \\
\text { Seater / Double Seater Rooms } \\
\text { Dining Hall \& Kitchen } \\
\text { Multipurpose Rooms } \\
\text { Activity Rooms }\end{array}$ & $\begin{array}{l}\text { Assuming capacity of new } \\
\text { hostel to be of } 500 \text { persons } \\
\text { consuming water @ } 135 \text { lpcd } \\
\text { then demand will be } \\
1.562 \mathrm{lps}\end{array}$ & $27 \mathrm{~m}$ \\
\hline 6 & $\begin{array}{l}\text { Badminton Court } \\
\text { Table Tennis }-2 \text { Tables } \\
\text { Chess, Carom } \\
\text { Reading Lounge } \\
\text { Outdoor Courts for Basket } \\
\text { Ball, Volley Ball }\end{array}$ & $\begin{array}{l}\text { Assuming Demand } \\
=0.82 \mathrm{lps}\end{array}$ & $12 \mathrm{~m}$ \\
\hline 7 & $\begin{array}{l}\text { No. of Flats : } 12 \text { to } 15 \text { with } \\
\text { Floor area of } 120 \text { Sq.m } \\
\text { Total No. of Flats }=70 \text { to } 85\end{array}$ & $\begin{array}{l}\text { Assuming } 72 \text { flats with } 6 \text { family } \\
\text { size consuming water @ } 150 \\
\text { lpcd, } \\
\text { Demand }=1.5 \mathrm{lps}\end{array}$ & $27 \mathrm{~m}$ \\
\hline
\end{tabular}

The above table 3 shows the water demand for new block.Net water demand (including auditorium, hostel, residential and floating water demand and new 7 blocks $)=27.68 \mathrm{lps}=2391552 \mathrm{lpd}$.

\section{RESULT: -}

Validation of results by Hardy Cross Method:

For approving outcomes given by EPANET, we should discover the release in pipes exclusively utilizing Hardy Cross Method and look at the two outcomes. On the off chance that EPANET results are near Hardy cross outcomes, at that point our product is 
getting us results which are legitimate as well. For this reason, we take circle close square 1 which comprises of 5 hubs system as appeared in figure 1.

According to EPANET results, pipes AB, BC, CD, DE and EA convey release of 0.65, 0.25, 10.08, 1.96 and 1.29. Presently will compute deviation (dQ) by strong cross strategy which ought to be exceptionally less, at that point just our results will be right.

$$
\underline{\sum R|Q| Q}
$$

Deviation is given as $\mathrm{dQ}=\frac{\sum 2 R Q}{\sum}$.

Taking loop direction in anticlockwise direction

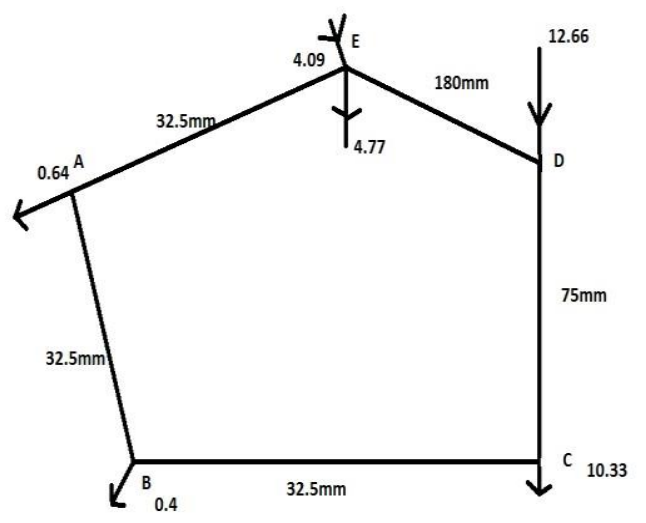

Figure 1: Hardy Cross Method

Table 4: Calculations for first trail

\begin{tabular}{|l|l|l|l|}
\hline Pipe & Radius R (in m) & $\begin{array}{c}\text { Discharge Q } \\
\text { in lps) }\end{array}$ & $\begin{array}{c}\sum R|Q| Q \\
\left(\mathbf{m}^{\wedge} \mathbf{7} \text { per sec) }\right.\end{array}$ \\
\hline $\mathrm{AB}$ & 0.0325 & 0.65 & $1.37 \mathrm{X} 10^{\wedge}-8$ \\
\hline $\mathrm{BC}$ & 0.0325 & 0.25 & $2.03 \mathrm{X} 10^{\wedge}-9$ \\
\hline $\mathrm{CD}$ & 0.075 & -10.08 & $-7.62 \mathrm{X} 10^{\wedge}-6$ \\
\hline $\mathrm{DE}$ & 0.090 & 1.96 & $1.76 \mathrm{X} 10^{\wedge}-7$ \\
\hline $\mathrm{EA}$ & 0.0325 & 1.29 & $5.4 \mathrm{X} 10^{\wedge}-8$ \\
\hline
\end{tabular}

$\mathrm{dQ}=-2.67 \mathrm{X} 10^{\wedge}-5 \mathrm{~m}^{\wedge} 3$ per sec $=-2.67 \times 10^{\wedge}-2$ lps which is very less so discharges flowing in pipes are true. Hence epanet results are valid. The above figure shows the calculation for first trail.

\section{CONCLUSION: -}

Subsequently here we are examined plan of water supply pipe networks. The point of the water supply organize framework is to guarantee that water achieves all zones in great quality and amount, with the end goal of investigation and reenactment to acquire least and most extreme power, speed of stream in the pipe arrange framework so as to guarantee the correct working of water pipe organize. Likewise, broke down circles organize as indicated by progression and vitality preservation equations because of the assurance of obscure releases, streams and weight at hubs. Strong Cross technique is utilized due to unravel and break down shut circles organize for stream coherence and head-misfortune so as to adjust the system as the setting of this paper.

[1] John Wiley et al., “"Design of Water Supply Pipe Networks” march 2008

[2] Arjun Kumar et al., "Design of Water Distribution System Usingepanet" (2015), Volume 3, Issue 9, 789 - 812

[3] seshagiri rao boddu et al.," DESIGN AND ANALYSIS OF PIPE NETWORK SYSTEM BY USING EPANET" March - May 2016

[4] Oluwaseun et al., "Design Analysis of Water Distribution Pipe Network Of Royal Valley Housing Estate In Ilorin Using Arc-Gis And Epanet" VOL. 3 NO. 2, DEC. 2017

[5] RASOOLI Ahmadullah et al., "Designing of Hydraulically Balanced Water Distribution Network Based on GIS and EPANET" Vol. 7, No. 2, 2016

[6] G. Anisha et al., "Analysis and Design of Water Distribution Network Using EPANET for Chirala Municipality in Prakasam District of Andhra Pradesh" ISSN: 2394-3661, Volume-3, Issue-4, April 2016

[7] Darshan Mehta et al., "Study of Water Distribution Network using epanet" march 2007

[8] Dagnachew Aklog et al.," All-in-one model for designing optimal water distribution pipe networks" 10.5194/dwes-10-33-2017

[9] Bragalli, C et al., On the optimal design of water distribution networks: A practical MINLP approach, Opt. Eng., 13, 219-246, 2012.

[10] K. Y., Seah et al., "Ant colony optimization for design of water distribution systems" Mgmt., 129, 200-209, 2003. 